\title{
Heavy Metal Pollution and Ecological Risk Assessment of Farmland Soil, Jilin Province
}

\author{
Fengxu Li ${ }^{1}$, Jiquan Zhang ${ }^{1, * *}$, Tiehua Cao ${ }^{2, * *}$, Xingpeng Liu ${ }^{1}, Z_{\text {Zhijun Tong }}{ }^{1}$, Yanan Chen ${ }^{1}$ \\ ${ }^{1}$ Institute of Natural Disaster Research, School of Environment, Northeast Normal University, \\ Changchun 130024, China \\ 2 Jilin Academy of Agricultural Sciences, Changchun 130033, China
}

\section{吉林省农田土壤重金属污染状况及 \\ 潜在生态风险评价 \\ 李丰旭 ${ }^{1}$, 张继权 ${ }^{1, *}$, 曹铁华 ${ }^{2, * *}$, 刘兴朋 ${ }^{1}$, 佟志军 ${ }^{1}$, 陈亚南 ${ }^{1}$ \\ ${ }^{1}$ 自然灾害研究所, 东北师范大学环境学院, 长春 130024 , 中国 \\ 2 吉林省农业科学院, 长春 130033 , 中国}

\begin{abstract}
122 soil samples were collected in farmland of Jilin Province, analyzed the pollution situation of 6 kinds of heavy metals by the pollution index method, the geoaccumulation index method and potential ecological risk. The results showed that: according to Nemero pollution index, there are $1.64 \%, 8.20 \%$ and $34.43 \%$ of soil samples in high level, moderate level and low level of pollution, respectively, but all is in low ecological risk. There is basically no pollution of $\mathrm{Zn}, \mathrm{Cu}, \mathrm{Hg}$ and $\mathrm{As}$ in farmland. The samples have $13.93 \%$ exceeding the second level $\mathrm{Cd}$ concentration of the national soil quality standard with average 1.65 times, mainly concentrated in Tonghua and Ji'an, southern Jinlin. The proportion of $\mathrm{Cd}$ ecological risk in moderate and considerable level is $3.28 \%$ and $9.84 \%$, respectively.
\end{abstract}

\footnotetext{
*第一通讯作者: 张继权 (1965-), 教授, 博士生导师, 主要从事环境灾害风险评价与管理、预警等方向研

究, 邮箱 zhangjq022@nenu. edu. cn

**第二通讯作者: 曹铁华 (1974-), 博士, 主要从事 农产品质量安全和生理生态研究, 邮箱: caotiehua2002@163.com
}

Keywords: Heavy metals; Pollution index method; Ecological risk assessment

摘要

采集吉林省农田土壤样品 122 个, 采 用污染指数法和潜在生态危害指数法分析 吉林省农田土壤中 6 种重金属污染状况。 结果表明: 吉林省农田土壤重金属污染具 有低生态危害，根据内梅罗综合污染指数 结果显示, 有 $1.64 \%$ 、8. $20 \%$ 和 $34.43 \%$ 土壤 样品分别处于重度污染、中度污染和轻度 污染水平; 样品中 $\mathrm{Zn} 、 \mathrm{Cu} 、 \mathrm{Hg}$ 和 $\mathrm{As}$ 基本 无污染; 土壤样品有 $13.93 \%$ 出现 Cd 超过国 家土壤二级质量标准, 平均超标 1.65 倍, 主要集中吉林省南部通化集安一带, $\mathrm{Cd}$ 生 态危害指数处于较重污染和中度污染的样 品比例分别为 $3.28 \%$ 和 $9.84 \%$ 。

关键词: 重金属; 污染指数法; 生态风险

\section{1. 引言}

土壤作为农业生产活动的基质, 同时 也是环境中污染物的一个 “汇”, 约有 $90 \%$ 的污染物最终汇集到土壤中 [1], 并且这些 污染物中有很大一部分持久性污染物。土 壤中重金属污染具有隐蔽性、滞后性、积 累性、不可逆性和难治理等特点, 重金属 
可迁移进入地下水, 被植物吸收进入食物 链 $[2-3]$, 对生态系统和人类身体健康具有 负效应 [4]。国内外针对重金属污染研究已 经有百年历史了 [5-7], 主要研究重金属空 间分布、污染等级评价和来源分析, 并且 对重金属污染现状进行风险评价, 以评估 重金属污染对生态环境和人类健康的危害。 其中, 生态风险评价被广泛应用到风险评 价研究中 [8-9], 用于评估土壤或沉积物中 污染物的生态环境效应 [10-11]。

拥有肥沃黑土地的吉林省作为中国农 业大省, 承担着的粮食生产任务, 2013 年 以 3551 万吨居中国粮食单产量第一 [12], 农田土壤安全更是关系着很大一部分人的 健康。虽然已有针对吉林省不同区域的农 田重金属污染现状调查[13-16], 但主要研 究局部农田土壤重金属含量和空间分布。

\section{2. 材料与方法}

\section{1 样品采集与分析}

在吉林省范围内如图 1 采取 122 个采 样点的农田土壤表层 0-20cm 混合样品, 将 土壤样品自然风干后过 100 目篮, 采用 HF$\mathrm{HNO}_{3}-\mathrm{HClO}_{4}$ 方法 [7] 消解土样, 利用石墨 炉原子吸收光谱仪测定消解液中的 $\mathrm{Zn} 、 \mathrm{Cu}$ 、 $\mathrm{Pb}$ 和 $\mathrm{Cd}$; 通过 $\mathrm{HNO}_{3}: \mathrm{HCl}(10 \mathrm{ml}, 1: 1 \mathrm{~V} / \mathrm{V})$ 消解样品 $2 \mathrm{~h}$, 采用原子荧光光谱仪测定土 壤中 $\mathrm{As}$ 和 $\mathrm{Hg}[1]$ 。所有重金属分析测试过 程采用国家一级标准物质 GBW07418GBW07422 (土壤) 进行质量控制, 回收率
为 $92.7 \%$ 114.9。数据经 $\operatorname{arcGIS} 10.0$ 和 Origin8. 0 处理分析, 进行统计特征和空间 分布特征描绘并评价吉林省农田土壤重金 属污染情况。

\section{2 评价方法}

污染指数评价方法采用单因子污染指 数法和内梅罗综合污染指数法评价吉林省 农田土壤重金属污染程度 [4]。一方面单因 子污染指数较全面的保留了数据的原始信 息, 计算公式:

$$
\mathrm{P}_{\mathrm{i}}=\mathrm{C}_{\mathrm{i}} / \mathrm{S}_{\mathrm{i}}
$$

其中, $\mathrm{Pi}$ 为重金属元素的单因子评价 指数; $\mathrm{Ci}$ 为重金属元素在土壤中的含量; $\mathrm{Si}$ 是重金属农田土壤质量标准, 即国家二 级环境质量标准值。

另一方面内梅罗综合污染指数法兼顾 了单因子污染指数的平均值和最高值, 能 较全面地反映土壤环境的总体质量 [1], 计 算公式如下:

$$
\mathrm{P}_{\text {综合 }}=\sqrt{\left(\left(\mathrm{Pi}_{\text {ave }}\right)^{2}+\left(P i_{\text {max }}\right)^{2}\right) / 2}
$$

式中, $P$ 综合为内梅罗综合污染指数; $\mathrm{Pi}_{\text {max }}$ 为所有污染物单因子污染指数中最大 值; $\mathrm{Pi}_{\mathrm{ave}}$ 为所有污染物单因子污染指数的平 均值。该方法对应的土壤环境质量评价分 级标准为: 当 $\mathrm{P}$ 综合 $\leq 1.0$ 为清洁的一级土壤; 当 $1.0<\mathrm{P}$ 综合 $\leq 2.0$ 为安全的二级土壤; 当 $2.0<\mathrm{P}$ 综合 $\leq 3.0$ 为警戒的三级土壤; 若 $\mathrm{P}$ 综合 $\geq 3.0$ 为污染的超三级土壤 [17]。

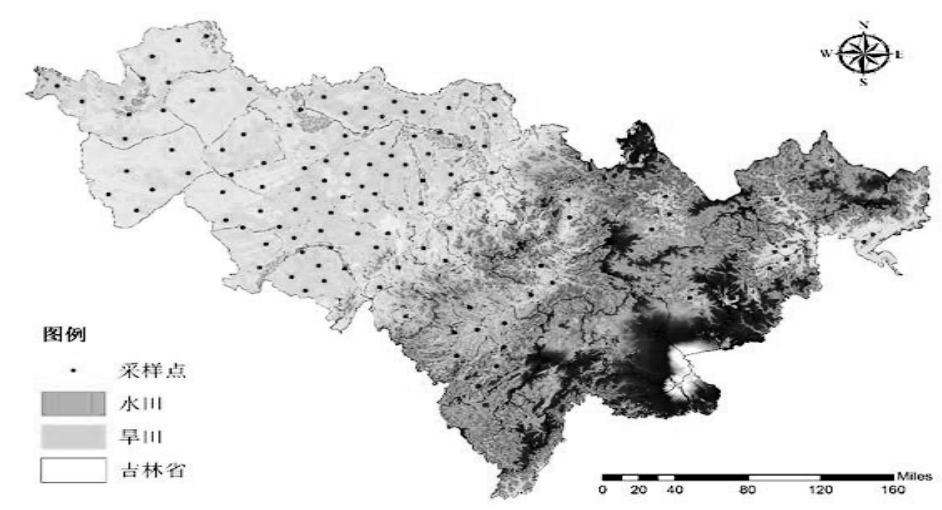

图 1 吉林省农田土壤采样点分布图 
潜在生态危害指数法综合考虑了重金 属的毒性在沉积物中普遍的迁移转化规律、 评价区域对重金属污染的敏感性以及重金 属区域背景值得差异, 可以综合反映沉积 物中重金属的潜在生态影响。计算公式如 下:

$$
\begin{gathered}
E_{r}^{i}=T_{r}^{i} \times C_{f}^{i}=T_{r}^{i} \times C_{s}^{i} / C_{n}^{i} \\
\mathrm{RI}=\sum E_{r}^{i}
\end{gathered}
$$

式中, $C_{s}^{i}$ 代表沉积物中重金属实测浓度, $\mathrm{mg} \cdot \mathrm{kg}^{-1} ; C_{n}^{i}$ 代表土壤中重金属背景参考值, $\mathrm{mg} \cdot \mathrm{kg}^{-1} ; T_{r}^{i}$ 代表单因子污染物毒性响应系 数, 本研究 $\mathrm{Zn} 、 \mathrm{Cu} 、 \mathrm{~Pb} 、 \mathrm{Cd} 、 \mathrm{Hg}$ 和 $\mathrm{As}$, 6 种重金属的 $T_{r}^{i}$ 值分别为 $1 、 5 、 5 、 30$ 、 40 和 10 。由于本研究只涉及 6 种重金属, 少于 Hakanson 提出的潜在生态风险指数法 时的 8 种, 因此采用刘文新等 [18]提出的

\begin{tabular}{|c|c|c|c|}
\hline$E_{r}^{i}$ 指数范围 & $\begin{array}{c}E_{r}^{i} \text { 指 } \\
\text { 数范 } \\
\text { 围 }\end{array}$ & $\begin{array}{c}\mathrm{RI} \text { 指数范 } \\
\text { 围 }\end{array}$ & $\begin{array}{l}\text { 程度 } \\
\text { 分级 }\end{array}$ \\
\hline$E_{r}^{i} \leq 30$ & 低度 & $\mathrm{RI} \leq 110$ & 低度 \\
\hline $30<E_{r}^{i} \leq 60$ & 中度 & $\begin{array}{c}110< \\
\mathrm{RI}<220\end{array}$ & 中度 \\
\hline $60<E_{r}^{i} \leq 120$ & 较重 & & \\
\hline $120<E_{r}^{i} \leq 240$ & 重度 & $\begin{array}{c}220< \\
\mathrm{RI}<440\end{array}$ & 重度 \\
\hline$E_{r}^{i}>240$ & 极重 & $\mathrm{RI}>440$ & 极重 \\
\hline
\end{tabular}
调整后的标准 (表 1 ) 进行评价。

\section{3. 结果与分析}

\section{1 农田土壤重金属污染水平及空间分布}

\section{特征}

122 个采样点土壤中的重金属 $\mathrm{Zn} 、 \mathrm{Cu}$ 、 $\mathrm{Pb} 、 \mathrm{Cd} 、 \mathrm{Hg}$ 和 $\mathrm{As}$ 含量, 参比 1990 年中国 环境监测总站发布的《中国土壤元素背景 值》中吉林省背景值和中国土壤质量标准 (GB15618-1995) 的二级标准（表 2)，6 种重金属的平均含量均低于国家二级标准, 并且偏度均大于零, 说明土壤中重金属浓 度大多数低于平均值, 只有少数高于平均 值, $\mathrm{Cu} 、 \mathrm{~Pb} 、 \mathrm{Cd} 、 \mathrm{As}$ 和 $\mathrm{Hg}$ 均属于中等变 异性 $(10 \%<\mathrm{Cv} \leq 100 \%)$ 而 $\mathrm{Zn}$ 是高变异性 $(\mathrm{Cv}>100 \%)$ [19], 说明大多数 6 种重金 属均存在局部高浓度, $\mathrm{Zn}$ 最显著, 这很可 能是由于地质构造造成该区域土壤母质就 具有较高 $Z n$ 含量 $[19]$ 。所以总体上吉林省 农田土受重金属污染并不严重, 只有局部 受 Cd 污染。

利用 GIS 分析技术进行空间分析。由 于克里金 (Kriging) 插值方法在数学上可 对所研究的对象提供一种最佳线性无偏估 计 (某点处的确定值) 的方法, 考虑到空 间相关性, 虽然原始的输入点可能会发生

\begin{tabular}{ccccccc}
\hline & 表 2 农田土壤重金属基本参数统计描述 & & & \\
\hline 项目 & $\mathrm{Zn}$ & $\mathrm{Cu}$ & $\mathrm{Pb}$ & $\mathrm{Cd}$ & $\mathrm{Hg}$ & $\mathrm{As}$ \\
\hline 平均值 $\left(\mathrm{mg} \cdot \mathrm{kg}^{-1}\right)$ & 21.72 & 15.09 & 46.08 & 0.2451 & 0.0378 & 4.957 \\
最小值 $\left(\mathrm{mg} \cdot \mathrm{kg}^{-1}\right)$ & 0.45 & 0.75 & 7.00 & 0.0050 & 0.0072 & 1.468 \\
最大值 $\left(\mathrm{mg} \cdot \mathrm{kg}^{-1}\right)$ & 127.60 & 91.37 & 118.72 & 1.1150 & 0.1600 & 12.919 \\
标准差 & 26.46 & 14.41 & 31.89 & 0.2169 & 0.0273 & 2.595 \\
变异系数 & 121.87 & 95.49 & 69.20 & 88.50 & 72.30 & 52.34 \\
偏度 & 1.59 & 2.44 & 0.53 & 1.79 & 2.13 & 0.65 \\
峰度 & 2.15 & 10.41 & -1.37 & 2.92 & 5.55 & -0.05 \\
吉林省背景值 $\left(\mathrm{mg} \cdot \mathrm{kg}^{-1}\right)$ & 72.8 & 16.4 & 27.9 & 0.0914 & 0.03 & 6.7 \\
国家二级标准 $\left(\mathrm{mg} \cdot \mathrm{kg}^{-1}\right)$ & 250 & 100 & 300 & 0.3 & 0.5 & 40 \\
\hline
\end{tabular}

变化, 但在数据点多时, 内插的结果可信 度较高。所以选用克里金空间插值方法绘 制重金属空间分布图 (图 2), Zn 高浓度主 要集中在东部, 但 $\mathrm{Zn}$ 最高浓度低于国家二 级标准; 在吉林矿产丰富的南端 $\mathrm{Cu}$ 浓度较 高, $\mathrm{Cu}$ 处于高出背景值, 低于国家二级标 准, 主要集中在拥有大面积葡萄种植区的 通化和集安市, 紧邻辽宁省, 很有可能来
源于含有 $\mathrm{Cu}^{2+}$ 的农药和杀虫剂的使用 [14]; 而 $\mathrm{Cd}$ 在扶余县超过了国家土壤质量标准的 二级标准, 大量研究显示, $\mathrm{Cd}$ 是农业实践 活动的标记元素, 主要由于农业生产中的 化肥使用 [7-12]; Hg 和 As 在经济发达且 人类活动居多的吉林省中部呈现高浓度水 平, As 仅有 1.64\%超过中国背景值但并不 超过国家二级标准, 而 $\mathrm{Hg}$ 高浓度主要集中 

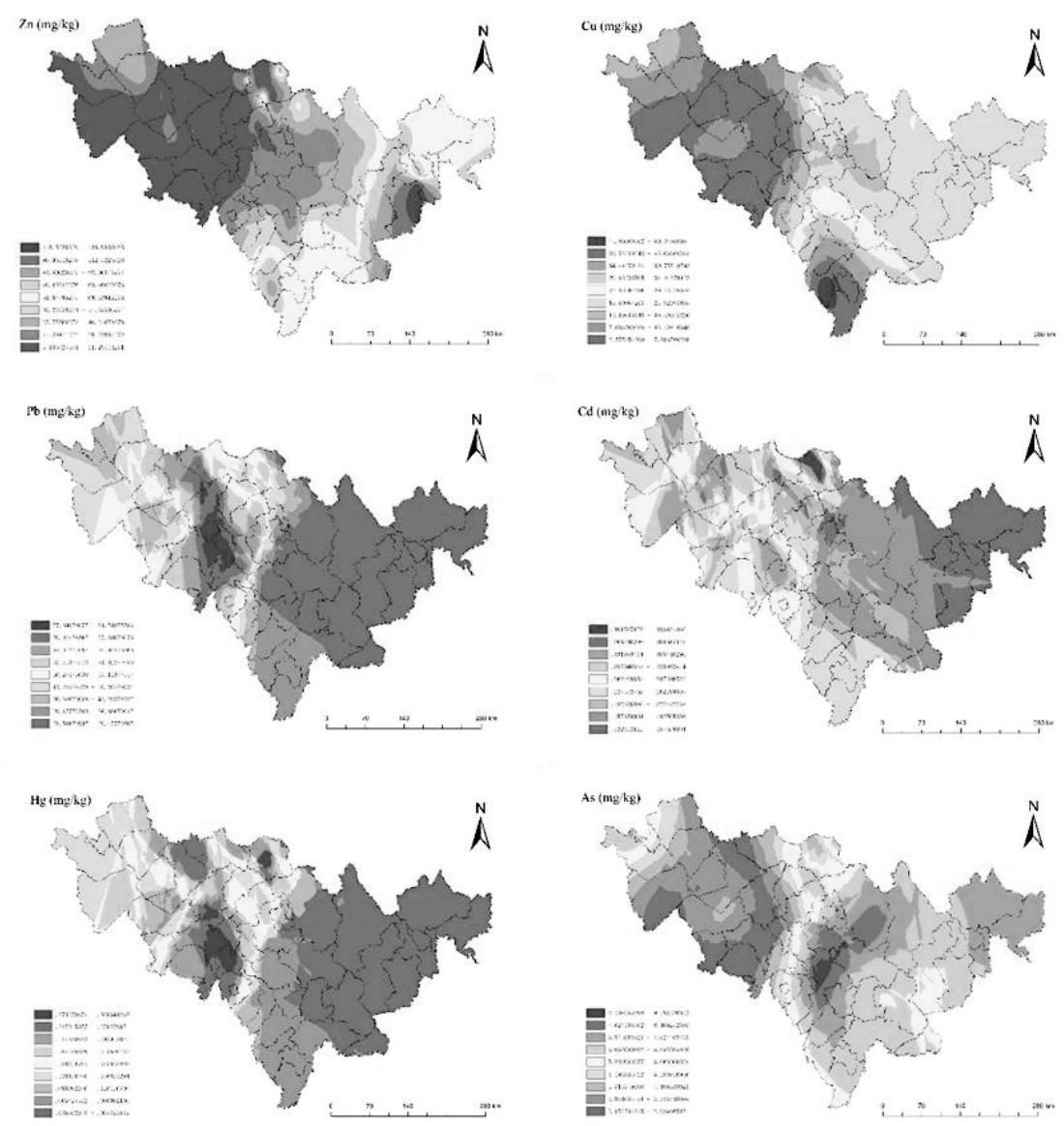

图 26 种重金属在吉林省农田土壤分布

在长春和吉林市, 但并不存在超标现象, 吉林中部长春、四平与松原之间浓度较高, 呈带状分布, 区域位置与吉林省黑土带分 布相近, 该区域也是吉林省经济发展中心 和工业中心, 也是贯穿南北交通运输干线 京哈高速和 102 国道必经之地。上个世纪 由于含 $\mathrm{Pb}$ 汽油的使用成道路两旁形成重金 属沉积 $[20]$ 。但 $\mathrm{Pb}$ 浓度仅是高于背景值, 并未超标。

\section{2 农田土壤重金属污染污染指数评价}

根据 6 种重金属单因子污染指数 Pi 绘 制箱形图 (图 3), 6 种重金属平均 $\mathrm{Pi}$ 值顺 序为 $\mathrm{Pb}>\mathrm{Cd}>\mathrm{Cu}>\mathrm{As}>\mathrm{Hg}>\mathrm{Zn}$ 。 $\mathrm{Pb}$ 的污
并且二者高浓度存在很大重叠区域; $\mathrm{Pb}$ 在 染单因子污染指数均值大于 1 并且上边缘 超过 3 , 但中位数小于 1 , 说明 $50 \%$ 以上为 清洁区, 少部分采样点处于 $\mathrm{Pb}$ 的重度污染 和中度污染, 并且污染指数大; $\mathrm{Cd}$ 于 $\mathrm{Pb}$ 的污染情况相似, $75 \%$ 地区处于轻度污染区 和清洁区, 存在少数采样点呈现 $\mathrm{Cd}$ 的中度 污染水平; $\mathrm{Zn} 、 \mathrm{Cu} 、 \mathrm{Hg}$ 和 $\mathrm{As}$ 的 $\mathrm{Pi}$ 值均小 于 1 , 表明吉林省农田土壤主要受 $\mathrm{Cd}$ 和 $\mathrm{Pb}$ 两种重金属污染, 这两种重金属元素是人 类活动引起污染的重要指示元素 [21]。结 合文献研究 [2-9, 14-16] 和空间分布特征, 推测中部地区 $\mathrm{Cd}$ 和 $\mathrm{Pb}$ 的污染除了来源于 化肥和农药外, $\mathrm{Cd}$ 可能源于农田污灌; $\mathrm{Pb}$ 
可能来源于汽车尾气粉尘沉降, 或者周边 工业废气排放。根据内梅罗综合污染指数, 吉林省农田土壤大约有 $1.64 \%$ 处于重度污染, 平, 所以吉林省农田土壤大部分不存在重 金属污染, 只在局部存在 $\mathrm{Pb}$ 和 $\mathrm{Cd}$ 的中度 污染。

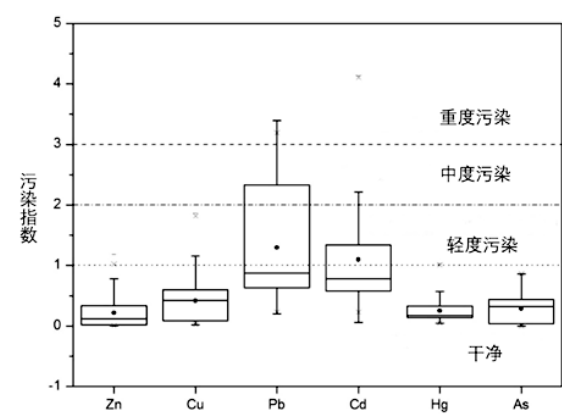

图 36 种重金属单因子污染指数箱形图

\section{3 农田土壤重金属污染潜在生态风险评}

\section{价}

农田土壤中重金属潜在生态风险系数 如图 4 所示, $\mathrm{Zn} 、 \mathrm{Cu} 、 \mathrm{~Pb} 、 \mathrm{Cd} 、 \mathrm{Hg}$ 和 $\mathrm{As}$ 的潜在生态危害指数均值均小于 30 , 属于 低度度生态危害, 其中 $\mathrm{Zn} 、 \mathrm{Cu} 、 \mathrm{~Pb} 、 \mathrm{Hg}$ 和 As 的最大危害指数都小于 30 , 说明在 研究区内, 这 5 种重金属只存在轻度潜在 生态危害, 对吉林省农田土壤的生态风险 比较低; 而 $\mathrm{Cd}$ 存在 $9.84 \%$ 和 3.28\%的样品 属于中度和较重生态危害, 对农田土壤存 在一定潜在生态风险, 是研究区域农田土 壤重金属生态风险的主要成因。由于本研 究选取 1990 年土壤普查为背景值, 该结果 也反映了吉林省农田土壤重金属污染减轻, 这与近年环境保护与规划管理实施有很大 关系, 减少各种形式重金属污染排放, 并 通过自然淋洗和作物重金属提取等途径自 净。所有采样点 RI 值小于 110 , 可在大尺 度下判定吉林省农田土壤重金属污染对生 态环境具有低度生态风险。

\section{4. 结论}

吉林省农田土壤中 $\mathrm{Zn} 、 \mathrm{Cu} 、 \mathrm{Hg}$ 和 $\mathrm{As}$ 均不存在污染现象, 人为造成的 $\mathrm{Pb}$ 中度污
另外, 大约 $8.20 \%$ 和 $34.43 \%$ 处于中度和轻 度污染水平, 所以吉林省农田土壤大部分 不存在重金属污染, 只在局部存在 $\mathrm{Pb}$ 和 $\mathrm{Cd}$ 染约占研究区域的 $19.67 \%$, 但并尚不存在 潜在生态危害风险; 在吉林省南部的辽源 和通化一带存在 $\mathrm{Cd}$ 超标现象, 具有较重程 度的潜在生态危害风险。对于 $\mathrm{Cd}$ 超标地区 应当采取措施, 减少污染排放, 进行合理 修复, 同时也要关注当地农作物 $\mathrm{Cd}$ 含量; 虽然黑土带目前未出现 $\mathrm{Pb}$ 超标现象, 但同 样要合理使用化肥, 避免加剧污染状况。

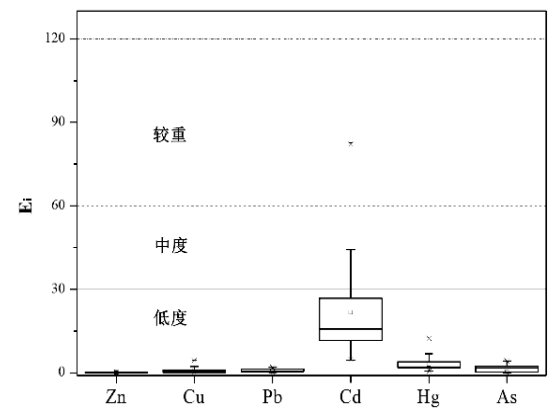

图 46 种重金属生态风险指数箱型图

\section{Acknowledgements}

This study was supported by the National Major Program of Water Pollution Control and Treatment Technology of China under Grant (NO. 2014ZX07201-011) and the National Non-Profit Research Program of China (NO.201401015)

\section{致谢}

本文以国家重大水专项（2014ZX07201-011） 和水利部公益性行业科研专项经费项目 （201401015）为项目支撑。

\section{参考文献}

[1] 韩平, 王纪华, 冯晓元, 等. 北京顺义区 土壤重金属污染生态风险评估研究. 农 业环境科学学报, 2015, 34(1): 103-109.

[2] Kumar Sharma R, Agrawal M, Marshall F. Heavy metal contamination of soil and vegetables in suburban areas of Varanasi, 
India. Ecotoxicology and Environmental Safety, 2007, 66(2): 258-266.

[3] Zhuang P, Mcbride M B, Xia H P, et al. Health risk from heavy metals via consumption of food crops in the vicinity of Dabaoshan mine, South China. Science of the Total Environment, 2009, 407(5): 1551-1561.

[4] Sultan K, Shazili N A. Distribution and geochemical baselines of major, minor and trace elements in tropical topsoils of the Terengganu River basin, Malaysia. Journal of Geochemical Exploration, 2009, 103(2-3), 57-68.

[5] Lin Y, Ma J, Zhang Z. et al. Linkage between human population and trace elements in soils of the Pearl River Delta: Implications for source identification and risk assessment. Sci. Total Environ. 2018, 610-611, 944-950..

[6] Yang P, Mao R, Shao H, Gao Y. An investigation on the distribution of eight hazardous heavy metals in the suburban farmland of China. Hazardous Mater. 2009, 167, 1246-1251.

[7] Ding M, Peng S, Mao L. et al. Major Element Geochemistry of LongShan Loess Profile in the Central Shandong Mountainous regions, Northern China. Journal of Risk Analysis and Crisis Response, Vol. 7, No. 3, 127-136.

[8] Zhang C, Yang Y, Li W, et al. Spatial distribution and ecological risk assessment of trace metals in urban soils in Wuhan, central China. Environmental Monitoring and Assessment, 2015, (187): 556.

[9] Hu B, Jia X, Hu J.et al. Assessment of Heavy Metal Pollution and Health Risks in the Soil-Plant-Human System in the Yangtze River Delta, China. Int. J. Environ. Res. Public Health. 2017, 14, 1042 .
[10] Ke X, Gui S, Huang H, et al. Ecological risk assessment and source identification for heavy metals in surface sediment from the Liaohe River protected area, China. Chemosphere, 2017(175): 473481.

[11] Ihedioha J N, Ukoha P O, Ekere N R. Ecological and human health risk assessment of heavy metal contamination in soil of a municipal solid waste dump in Uyo, Nigeria. Environmental Geochemistry and Health, 2016: 1-19.

[12] Ma X, Ma Y. The spatiotemporal variation analysis of virtual water for agriculture and livestock husbandry: A study for Jilin Province in China. Science of the Total Environment, 2017, 586: 1150-1161.

[13] Chai Y, Guo J, Chai S, et al. Source identification of eight heavy metals in grassland soils by multivariate analysis from the Baicheng-Songyuan area, Jilin Province, Northeast China. Chemosphere, 2015, 134: 67-75.

[14] 刘艳军.吉林省重金属污染现状调查.资 源与环境,2012(20):34.

[15] 梁烜赫, 曹铁华, 张否,等. 吉林省农田重 金属含量及其在作物中的积累. 吉林农 业科学, 2011, 36 (6): 59-62.

[16] 唐丽静, 王冬艳, 李月芬, 等. 吉林黑土 区土壤 - 作物系统重金属元素地球化 学特征研究. 山东农业大学学报 (自然 科学版), 2014, 45 (1): 136-143.

[17] Ihedioha J N, Ukoha P O, Ekere N R. Ecological and human health risk assessment of heavy metal contamination in soil of a municipal solid waste dump in Uyo, Nigeria. Environmental Geochemistry and Health, 2016: 1-19.

[18] Yang Y, Christakos G, Guo M, et al. Space-time quantitative source apportionment of soil heavy metal concentration increments. Environmental Pollution,2017(223): 560-566. 
[19] 刘文新, 奕兆坤, 汤鸿霄. 乐安江沉积物 中重金属元污染的潜在生态风险评价. 生态学报, 1999, 19(2): 206-211.

[20] Li F, Zhang J, Cao T. et al. Human Health Risk Assessment of Toxic Elements in Farmland Topsoil with Source Identification in Jilin. Province, China. Int. J. Environ. Res. Public Health. 2018, $15,1040$.

[21] 方晓波, 史坚, 廖欣峰, 等. 临安市雷竹 林土壤重金属污染特征及生态风险评 价. 应用生态学报. 2015(6), 26,6:18831891. 